\title{
Comparison of coronary risk scoring systems to predict the severity of coronary artery disease using the SYNTAX score
}

\author{
Hatice Tolunay ${ }^{1}$, Ozge Kurmus ${ }^{2}$ \\ ${ }^{1}$ Clinic of Cardiology, Abdurrahman Yurtaslan Ankara Oncology Education \\ and Research Hospital, Ankara, Turkey \\ ${ }^{2}$ Clinic of Cardiology, Mersin State Hospital, Ankara, Turkey
}

\begin{abstract}
Background: Reliable risk scoring systems that can predict the severity of coronary artery disease (CAD) are necessary to implement effective management strategies in high-risk patients. Atherosclerotic vascular disease and with cardiovascular morbidity and mortality risk are patients who would benefit most from a change in patients at high risk factors. Framingham Risk Score, the Prospective Cardiovascular Münster (PROCAM) score and Systematic Coronary Risk Evaluation (SCORE) risk scores are used for this purpose. The severity of the CAD as detected by coronary angiography can be estimated using the SYNTAX score. In this study, it is aimed to assess the relation between SYNTAX score and the Framingham, PROCAM and SCORE scores in the context of their utility for the determination of the severity of the CAD.

Methods: A total of 205 patients with documented CAD who underwent coronary angiography due to a diagnosis of stable angina pectoris were included in this study. Coronary risk scores were determined for each patient. The relation between the SYNTAX score and the results of coronary risk scoring systems were analyzed.

Results: A positive relationship between the SYNTAX score, which reflects the severity of the $C A D$ and coronary risk scores was found. However, Framingham and SCORE were superior, i.e., had more predictive value, regarding their ability to predict the SYNTAX score $(p=0.029$, 0.033 and 0.002 , respectively).

Conclusions: Examination of the distribution of SYNTAX score across low, intermediate and high-risk groups showed a significantly higher predictive value of SCORE for high-risk patients ( $p=0.005)$. (Cardiol J 2016; 23, 1: 51-56)
\end{abstract}

Key words: Framingham, PROCAM, SCORE, SYNTAX

\section{Introduction}

Risk estimation based on the results of reliable risk prediction tools such as Framingham Risk Score (FRS), Systematic Coronary Risk Evaluation (SCORE), and the Prospective Cardiovascular Münster (PROCAM), followed by lifestyle changes and medical management are the main targets in the prevention of coronary artery disease (CAD).

Framingham risk scoring system takes a number of different factors into account such as age, smoking status, blood pressure (BP), total cholesterol and high density lipoprotein (HDL) cholesterol in order to provide an age- and sex-matched

Address for correspondence: Hatice Tolunay, MD, Park caddesi, Atabilge sitesi 36/16, 06200 Cayyolu, Ankara, Turkey, tel: +903122408527, e-mail: drhaticearslan@gmail.com

Received: 24.07.2015 Accepted: 15.10.2015 
risk comparison for the 10-year risk of myocardial infarction (MI) and cardiovascular mortality. Low, intermediate, and high risk groups are defined on the basis a risk level below $10 \%$, between $10 \%$ and $20 \%$, and above $20 \%$, respectively [1].

PROCAM algorithm allows the estimation of 10-year acute coronary event (MI and sudden cardiac death) risk using a number of variables such as age, gender, systolic BP, HDL, low density lipoprotein, triglycerides, diabetes mellitus, smoking status, and family history [2]. The calculated 10 -year coronary risk is considered low if it is $<10 \%$, moderate if it is between $10 \%$ and $20 \%$, or high if it is $>20 \%$.

On the other hand, SCORE risk algorithm uses the following variables to yield a 10 -year cardiovascular death risk: age, gender, smoking, systolic BP, total cholesterol, and low or high local risk group. Presence of risk factors and the cardiovascular disease may change in time and can also vary for different societies. The SCORE risk scale is prepared for high and low risk groups by taking into consideration the mortality statistics of various countries.

The difference between SCORE and FRS is that the former does not only predict the risk of the development of $\mathrm{CAD}$, but also the risk of dying due to cardiovascular disease. As shown by the SCORE algorithm, Turkey is considered a high risk geographical area. The 10-year cardiovascular mortality risk is low, intermediate, or high if it is $<0 \%$ to $4 \%$, between $5 \%$ and $9 \%$, and above $10 \%$, respectively [3].

SYNTAX score is used to estimate the extent and severity of the CAD through the assessment of the number of angiographically detected coronary lesions, their functional effects, locations, and complexity. The following variables are taken into consideration for SYNTAX score estimates: coronary dominance, location at bifurcation, trifurcation, or ostial lesions, tortuosity, calcifications, the content of the thrombus, presence of diffuse disease, and elongated lesions. This tool provides guidance on the appropriate revascularization technique to be used [4].

Practical and reliable parameters are required to estimate the presence and severity of CAD and to implement effective diagnostic and therapeutic strategies. In this study, we examined the usefulness of CAD risk scoring systems in the abovementioned context and assessed their correlations with the SYNTAX score.

\section{Methods}

This study was designed as a retrospective study, which was performed in the Cardiology Department of Abdurrahman Yurtaslan Ankara Oncology Education and Research Hospital. A total of 205 patients with significant CAD who underwent a coronary angiography due to a diagnosis of stable angina pectoris between January 2012 and December 2014 were included in this study.

Local Ethics Committee approved the study and written consent was taken from the study participants. Of patients who had undergone coronary angiography in different centers, coronary risk factors were examined and coronary angiography SYNTAX scores are calculated based on the evaluation report. Standard physical examination was performed and the age, height, weight, systolic and diastolic BP, history of hypertension and/or diabetes, a family history of CAD, smoking status, body mass index and lipid profile (in fasting blood samples) were determined. Hypertension was defined as systolic BP $>140 \mathrm{~mm} \mathrm{Hg}$ or a diastolic BP $>90 \mathrm{~mm} \mathrm{Hg}$, or use of antihypertensive medications. A positive family history for $\mathrm{CAD}$ was defined as evidence of CAD, such as a history of MI, coronary artery bypass surgery. Framingham Risk Score, PROCAM, and SCORE coronary artery disease risk scores were calculated for each patient. Using the FRS system, two different risk scores were calculated i.e. a 10-year coronary event risk score and age- and sex-matched comparative Framingham Risk Score (C-FRS). Exclusion criteria were unstable angina, previous MI, previous history of percutaneous or surgical myocardial revascularization, contrast allergy and renal failure. Since patients above 65 years of age are not included in the assessment for SCORE risk level, patients older than 65 years of age and patients receiving lipid-lowering therapy were excluded from the study.

Coronary angiography results were evaluated by another researcher from the study team and SYNTAX scores were calculated to estimate the extent and severity of the CAD. A narrowing greater than $50 \%$ in a vessel with a diameter exceeding $1.5 \mathrm{~mm}$ was considered a significant lesion. SYNTAX scores are calculated and the prognostic value of the SYNTAX score in relation to cardiovascular events, which were defined as low-risk (SYNTAX score 0-22), intermediate-risk (SYNTAX score 23-32) and high-risk (SYNTAX score > 32) was determined. 
The correlation between coronary artery risk scores as determined by FRS, PROCAM, and SCORE systems and the severity and extent of the CAD as determined by the SYNTAX score was evaluated.

\section{Statistical methods}

SPSS 15.0 software package was used for statistical analyses. Parametric variables were expressed as mean \pm standard deviation and categorical variables were expressed as percentage (\%). Before analyzing the association between the SYNTAX score and FRS, SCORE, and PROCAM scores, Kolmogorov-Smirnov test was performed for normality hypothesis. As the normality hypothesis was accepted for 3 variables, Pearson's coefficient was used as the association coefficient. This association was confirmed using linear regression analysis. A p-value less than 0.05 was considered statistically significant. The distribution of SYNTAX scores across risk Groups (low, intermediate, high) was analyzed with analysis of variance (ANOVA) and their distribution is shown as mean values and standard deviations in the graph.

\section{Results}

The mean age of the participants $(n=205)$ was $58.00 \pm 6.58$ years, with male and female patients representing $57.3 \%$ and $42.7 \%$ of the total study population, respectively. Overall, $72.7 \%$ of the patients had hypertension, $32.2 \%$ had diabetes, $20.5 \%$ had positive family history, and $50.7 \%$ had hyperlipidemia. The baseline patient characteristics are summarized in Table 1.

The mean FRS was $16.54 \pm 10.04$, the comparative FRS was $17.12 \pm 6.78$, the mean PROCAM score was $18.26 \pm 14.67$, the mean SCORE was $6.61 \pm 4.43$ and the mean SYNTAX score was $13.63 \pm 8.68$. The risk scoring data for CAD are shown in Table 2. Using the SYNTAX score, the patients were categorized into three groups according
Table 1. Baseline demographic characteristics ( $n=205)$.

\begin{tabular}{lc}
\hline Age $[$ years] & $58.00 \pm 6.58$ \\
Total cholesterol $[\mathrm{mg} / \mathrm{dL}]$ & $203.42 \pm 40.12$ \\
LDL $[\mathrm{mg} / \mathrm{L}]$ & $126.80 \pm 38.25$ \\
Triglyceride $[\mathrm{mg} / \mathrm{dL}]$ & $192.89 \pm 85.76$ \\
$\mathrm{HDL}[\mathrm{mg} / \mathrm{dL}]$ & $40.41 \pm 10.55$ \\
Body mass index $\left[\mathrm{kg} / \mathrm{m}^{2}\right]$ & $27.71 \pm 4.89$ \\
Gender (woman) & $42.7 \%$ \\
Hypertension & $72.7 \%$ \\
Diabetes mellitus & $32.2 \%$ \\
Hyperlipidemia & $50.7 \%$ \\
Cigarette & $51.7 \%$ \\
Family history & $20.5 \%$ \\
\hline
\end{tabular}

Data are presented as number (percentage) and mean \pm standard deviation; Adjusted for body mass index, family history of myocardial infarction, before the age of 60 years, history of past or current cholesterol treatment at baseline, and randomized treatment assignment. Hypertension was defined as self-reported systolic blood pressure of $140 \mathrm{~mm} \mathrm{Hg}$, or diastolic blood pressure of $90 \mathrm{~mm} \mathrm{Hg}$, or current antihypertensive medication regardless of blood pressure. Current cigarette smoker for a certain period of last year, which was defined as smoking history; LDL — low density lipoprotein; HDL — high density lipoprotein

to the Myocardial Intervention Guideline as proposed by the European Society of Cardiology. There were a total of $162(79 \%), 32(15.6 \%)$ and $11(5.4 \%)$ patients with a SYNTAX score between 1 and 22, 23 and 32 , and $>32$, respectively.

In patients with a low risk score for CAD, the percentages of individuals with a SYNTAX score $>32$ in FRS, C-FRS, PROCAM, and SCORE were $6.3 \%, 6.1 \%, 5.6 \%$, and $5.3 \%$, respectively; in contrast, among subjects with a high risk score, the corresponding percentages of individuals with a SYNTAX score $<22$ were $73.5 \%, 74.8 \%, 78.6 \%$, and $63 \%$, respectively.

The association between the risk scores and the SYNTAX score for the severity of CAD was explored. A significant association between SYNTAX score and SCORE $(p=0.002)$, FRS $(p=0.029)$,

Table 2. The percentages of low, intermediate or high risk groups and the average values.

\begin{tabular}{lcccc}
\hline & Low risk & Intermediate risk & High risk & Mean score \\
\hline FRS & $64(31.2 \%)$ & $92(44.9 \%)$ & $49(23.9 \%)$ & $16.54 \pm 10.04$ \\
C-FRS & $21(10.2 \%)$ & $115(56.1 \%)$ & $69(33.7 \%)$ & $17.12 \pm 6.78$ \\
PROCAM & $71(34.6 \%)$ & $64(31.2 \%)$ & $70(34.1 \%)$ & $18.26 \pm 14,67$ \\
SCORE & $76(37.1 \%)$ & $83(40.5 \%)$ & $46(22.4 \%)$ & $6.61 \pm 4.43$ \\
SYNTAX & $162(79 \%)$ & $32(15.6 \%)$ & $11(5.4 \%)$ & $13.63 \pm 8.68$ \\
\hline
\end{tabular}

Number (percentage) of patients with low, intermediate and high risk score; FRS — Framingham Risk Score; C-FRS — comparative FRS; PROCAM - Prospective Cardiovascular Münster; SCORE — Systematic Coronary Risk Evaluation 
Table 3. The association between the risk scores and the SYNTAX score.

\begin{tabular}{llcccc}
\hline & & FRS & C-FRS & PROCAM & SCORE \\
\hline \multirow{2}{*}{ SYNTAX } & Pearson correlation & $0.152^{*}$ & $0.149^{*}$ & 0.114 & $0.216^{* *}$ \\
& Sig. (2-tailed) & 0.029 & 0.033 & 0.104 & 0.002 \\
& N & 205 & 205 & 205 & 205 \\
\hline
\end{tabular}

${ }^{*}$ Correlation is significant at the level of 0.05 (2-tailed); ${ }^{*}$ Correlation is significant at the level of 0.01 (2-tailed); abbreviations as in Table 2

Table 4. Mean SYNTAX score comparison in low, intermediate or high risk groups with linear regression analysis.

\begin{tabular}{lccc}
\hline & B (regression coefficient) & SD & P \\
\hline Model constant & 11.252 & 1.651 & $<.001$ \\
FRS & 0.055 & 0.090 & 0.540 \\
C-FRS & -0.076 & 0.147 & 0.605 \\
PROCAM & -0.021 & 0.055 & 0.702 \\
SCORE & 0.477 & 0.221 & 0.032 \\
\hline
\end{tabular}

SD — standard deviation; rest abbreviations as in Table 2

and comparative FRS ( $\mathrm{p}=0.033)$ scores was found. Our results suggest that a risk categorization based on SCORE, FRS and C-FRS results is a more reliable indicator of the severity of the $\mathrm{CAD}$ as compared to PROCAM ( $\mathrm{p}=0.104)$ (Table 3 ). This association was confirmed by the linear regression analysis.

Linear regression analysis was used to perform a SYNTAX score comparison in low, moderate, or high risk groups defined on the basis of FRS, C-FRS, PROCAM, and SCORE. SYNTAX score was included as a dependent variable in the model. The significance level of the model is $p=0.038$. According to SYNTAX scores, SCORE risk estimation system showed a significant superiority over other variables $(\mathrm{p}=0.032)$ (Table 4$)$.

The distribution of SYNTAX score among low, intermediate and high risk groups in other risk estimation tools was examined and a significant difference for SCORE was found in the high-risk SYNTAX score group $(\mathrm{p}=0.005)$ (Fig. 1$)$.

\section{Discussions}

Scoring systems utilized to estimate the risk of cardiovascular events also provide an important means for estimating the presence and severity of coronary arteriosclerosis. Determination of highrisk individuals may facilitate the process whereby modifiable risk factors can be controlled in order to prevent coronary heart disease. In conjunction with

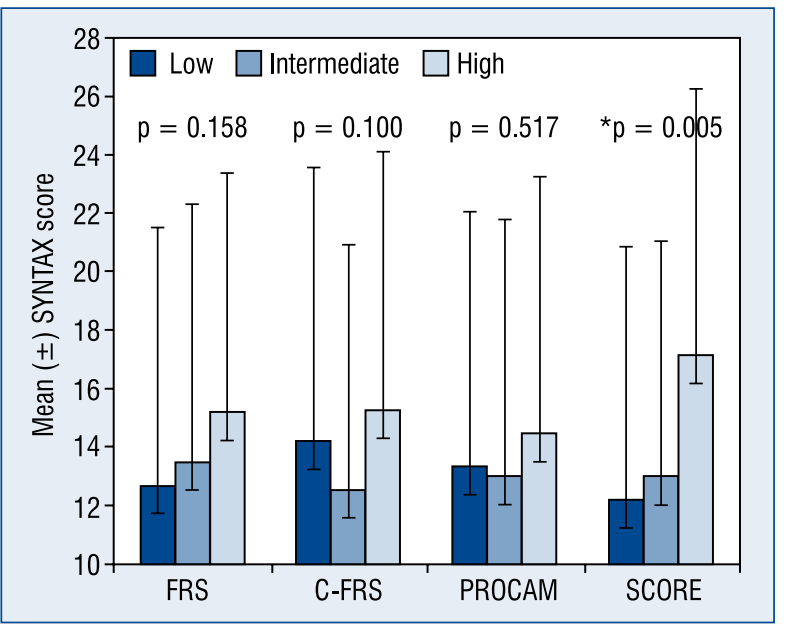

Figure 1. The distribution of SYNTAX score in low, intermediate and high risk groups as defined by Framingham Risk Score (FRS), comparative Framingham Risk Score (C-FRS), the Prospective Cardiovascular Münster (PROCAM) or Systematic Coronary Risk Evaluation (SCORE); ${ }^{*}$ Correlation is significant at the level of 0.05 .

the increase in average lifespan and cardiovascular disease incidence in today's world, clinical risk profile represents a first step in the assessment of patients. An effective risk assessment will certainly allow more efficient use of diagnostic tools, especially when one considers the accompanying costs and disabilities associated with these conditions. 
In the present study, the association between the SYNTAX score, which reflects the extent and severity of the $\mathrm{CAD}$, and other risk estimation tools for estimating the risk of cardiovascular death and MI were explored. Our results suggest that a risk categorization based on SCORE, FRS and C-FRS results is a more reliable indicator of the severity of the CAD as compared to PROCAM. PROCAM, FRS and SCORE risk scores seem to be barely useful in a secondary prevention setting. In patients with $\mathrm{CAD}$ high risk group, the cardiovascular risk seems to be better assessed by means of the SCORE risk score.

Literature provides many studies examining the relation between CAD and FRS. In some of these studies, calcified and non-calcified plaque burden and coronary artery calcium score (CACS) were evaluated in the coronary tomography angiography (CTA) [5-7]. It has been highlighted that specifically for intermediate risk groups for which clinical decision making is difficult, FRS should be supported by additional data like CACS [5].

The association between FRS and other clinical predictors with Total Plaque Score was examined by CTA and coronary atherosclerosis was present in the majority of patients with medium and low probability. In a small number of high risk patients were shown to have no evidence of atherosclerosis [8].

One previous study that investigated the association between left coronary system plaque progression and FRS, PROCAM, and SCORE results using intravascular ultrasound found highest correlation between PROCAM score and plaque progression [9].

In a study involving 1,296 patients undergoing a CTA with a diagnosis of stable CAD, Framingham and SCORE risk assessment tools were found to be superior as compared to PROCAM and Diamond Forrester scoring systems [10]. FRS high risk category included less patients with significant CAD and events, compared to patients with high risk using PROCAM, SCORE or Diamond Forrester in this study. In our study, there was a lower proportion of patients with a low coronary artery risk score and high SYNTAX score, while a significantly higher number of patients had high risk score with low SYNTAX score. Our results are similar with the study which has been made by Versteylen et al. [10]. Both studies also include patients with stable angina pectoris and in the same age group. This conclusion is due to the fact that patients over 65 and the patients with acute coronary syndrome were not involved in the study [10-14].
In a previous study examining the association between SYNTAX scores and serum uric acid level, smoking status, and FRS, a significant association between FRS and SYNTAX was found $(\mathrm{p}<0.001)$, whereas a significant association was found between SYNTAX and FRS ( $p=0.029)$ and age- and sex-matched comparative FRS ( $p=0.033$ ) [11].

In other ones, severity and extent of the CAD were evaluated in the coronary angiography with different methods [12, 13]. Each other scoring tools for the estimation of the severity of the CAD is the Jenkins' scoring [12] and Gensini scoring systems [13].

In a study by Sayın et al. [13] examining the utility of the FRS for the assessment of the severity of the CAD, a significant $(\mathrm{p}<0.0001)$ association between FRS and the Gensini score was observed.

In another study comparing the risk scoring systems for coronary arteriosclerosis and cardiovascular risk, coronary arteriosclerosis was classified as "single vessel", "two vessel" and "three vessel" disease. In that study, the value of FRS, modified FRS, PROCAM, and SCORE in evaluating the presence and severity of the CAD was examined, with a higher predictive value for FRS [14].

Correlation analyses showed a superiority of FRS, C-FRS, and SCORE as compared to PROCAM, and SCORE was particularly more capable of predicting the extent of the CAD in high risk patients. Our study is the first of its kind in terms of a comparison between SCORE results and risk estimation tools.

Previous studies have examined the usefulness of individual coronary risk scoring systems in estimating the presence, extent, and severity of stable CAD. In these studies, while the 10-year cardiovascular risk score was used for FRS estimations, the age- and sex-matched comparative risk scores were disregarded. Also, in contrast with a limited number of studies looking at the combined use of FRS and SYNTAX, our study was the first of its kind that assesses the association between the SYNTAX score and the results of PROCAM and SCORE risk estimation tools.

Cardiovascular risk scores correlate with the severity and extent of the CAD. In this regard SCORE, FRS, and C-FRS represent the most reliable risk assessment tools available. In our view, our findings may be particularly useful for re-assessing the value of other scoring systems, or for choosing the most appropriate risk assessment tool for cardiovascular disease screening. Also, the use of such clinical scoring systems bears important clinical significance with regard 
to the determination of individuals with a high risk of coronary arteriosclerosis and for preventing unnecessary invasive procedures through the implementation of lifestyle measures before the development of symptoms.

\section{Limitations of the study}

There are several limitations to our study. Patients with a previous history of revascularization due to $\mathrm{CAD}$, patients presenting with a diagnosis of acute coronary syndrome and patients currently receiving treatment due to hyperlipidemia were excluded from the study. FRS and C-FRS coronary risk scores relations with SYNTAX score is extremely weak, although the p value was significant. Even though SCORE risk scores relations with SYNTAX score is significant, patients under 40 or over 65 years of age were not included in the study, since SCORE risk assessment was not performed in these patients. Risk scoring system used in this study are not specific to Turkish population. Therefore coronary risk scoring systems should be supplemented by country-specific data.

\section{Conclusions}

In patients with stable angina pectoris, FRS, age and sex-matched comparative FRS, and SCORE risk assessment tools have been found to have superior predictive value than PROCAM for the prediction of the CAD. SCORE appears to be the most reliable risk assessment tool for predicting the severity and extent of the CAD, especially in terms of CAD in high risk groups.

Our findings suggest that among the risk scoring tools used for the assessment of CAD risk, SCORE distinguishes itself with its ability to provide reliable guidance on the presence, extent and severity of CAD. However, exclusion of diabetes, which is an important cardiovascular risk factor, and of patients over 65 years of age are important limitations of this method. Therefore, reliable risk estimation tools also addressing these drawbacks are warranted.

Conflict of interest: None declared

\section{References}

1. D'Agostino RB Sr, Vasan RS, Pencina MJ et al. General cardiovascular risk profile for use in primary care: The Framingham Heart Study. Circulation, 2008; 117: 743-753.

2. Assmann G, Cullen P, Schulte H. Simple scoring scheme for calculating the risk of acute coronary events based on the 10-year follow-up of the prospective cardiovascular Munster (PROCAM) study. Circulation, 2002; 105: 310-315.

3. Conroy RM, Pyorala K, Fitzgerald AP et al. Estimation of tenyear risk of fatal cardiovascular disease in Europe: The SCORE project. Eur Heart J, 2003; 24: 987-1003.

4. Sianos G, Morel MA, Kappetein AP et al. The SYNTAX Score: an angiographic tool grading the complexity of coronary artery disease. EuroIntervention, 2005; 1: 219-227.

5. Greenland P, LaBree L, Azen SP, Doherty TM, Detrano RC. Coronary artery calcium score combined with Framingham score for risk prediction in asymptomatic individuals. JAMA, 2004; 291: 210-215.

6. DeFilippis AP, Blaha MJ, Ndumele CE et al. The association of Framingham and Reynolds risk scores with incidence and progression of coronary artery calcification in MESA (Multi Ethnic Study of Atherosclerosis). J Am Coll Cardiol, 2011;58:2076-83.

7. Schneer S, Bachar GN, Atar E, Koronowski R, Dicker D. Evaluation of Framingham and systematic coronary risk evaluation scores by coronary computed tomographic angiography in asymptomatic adults, Am J Cardiol, 2013;111:700-4.

8. Pen A, Yam Y, Chen L, Dennie C, McPherson R, Chow BJW. Discordance between framingham risk score and atherosclerotic plaque burden. Eur Heart J, 2013; 34: 1075-1082.

9. Kim SW, Mintz GS, Escolar E et al. The impact of cardiovascular risk factors on subclinical left main coronary artery disease: An intravascular ultrasound study. Am Heart J, 2006; 152: 693.e7-12.

10. Versteylen MO, Joosen IA, Shaw LJ, Narula J, Hofstra L. Comparison of Framingham, PROCAM, SCORE, and Diamond Forrester to predict coronary atherosclerosis and cardiovascular events. J Nucl Cardiol, 2011; 18: 904-911.

11. Xiong Z, Zhu C, Qian X, Zhu J, Wu Z, Chen L. Predictors of clinical SYNTAX score in coronary artery disease: Serum uric acid, smoking and Framingham risk stratification. J Invasive Cardiol, 2011; 23: 501-504.

12. Kaulgud RS, Pradeep N, Kumbhar DP et al. Coronary heart disease Risk Scores and their correlation with Angiographic Severity Scores. Int J of Biomed Res, 2013; 4: 257-263.

13. Sayin MR, Cetiner MA, Karabag T et al. Framingham risk score and severity of coronary artery disease. Herz, 2013; $21: 21$.

14. Yalcin M, Kardesoglu E, Aparci M et al. Cardiovascular risk scores for coronary atherosclerosis. Acta Cardiol, 2012; 67: 557-563. 